\title{
$\omega$ structure in steel: a first-principles study
}

\author{
Yuji Ikeda ${ }^{a}$, Isao Tanaka ${ }^{a, b, c, d}$ \\ ${ }^{a}$ Center for Elements Strategy Initiative for Structure Materials (ESISM), Kyoto University, Kyoto 606-8501, Japan \\ ${ }^{b}$ Department of Materials Science and Engineering, Kyoto University, Kyoto 606-8501, Japan \\ ${ }^{c}$ Center for Materials Research by Information Integration, National Institute for Materials Science (NIMS), Tsukuba 305-0047, Japan \\ ${ }^{d}$ Nanostructures Research Laboratory, Japan Fine Ceramics Center, Nagoya 456-8587, Japan
}

\section{Abstract}

Recent experimental works reported observation of the $\omega$ structure in steel. Here, stability of the $\omega$ structure in steel is investigated based on first-principles with special interests in effects of interstitial C atoms. The interstitial $\mathrm{C}$ atoms increase the energy of the $\omega$ structure compared with the ferromagnetic (FM) BCC. The $\omega$ structure incorporating $\mathrm{C}$ atoms is also mechanically unstable unless the $\mathrm{C}$ concentration is 25 at.\%. It is concluded that the $\omega$ structure is mostly unstable in steel, and the $\omega$ structure in steel may be formed under special atomic constraints at twin boundaries or other interfaces.

Keywords: First-principles calculations, $\omega$ structure, Steel

\section{Introduction}

Nanometer-size domains of the $\omega$ structure have recently been reported in steel, i.e., Fe-C-based alloys, by detailed transmission electron microscopy observations $[1,2]$. This motivated us to investigate the stability of the $\omega$ structure in steel. The present authors' group has emphasized the importance of the $\omega$ structure on transformation between the BCC and the FCC structures in metallic systems. Togo and Tanaka developed a search algorithm for transformation pathways based on a systematic set of first-principles calculations and revealed that the $\omega$ structure was located on a transformation pathway between the BCC and the FCC structures [3]. Ikeda et al. suggested that the pressure-induced phase transition between the BCC and the FCC Fe at high temperature occurred along this transformation pathway [4]. The present authors recently performed a systematic investigation into the $\omega$ structure of transition elements [5]. The elemental $\omega$ Fe with antiparallel magnetic moments (+- - magnetic state) was found to be the lowest in energy among the investigated magnetic states and to be mechanically stable. - The $+--\omega \mathrm{Fe}$ was, however, $170 \mathrm{meV} /$ atom higher in energy than the ferromagnetic (FM) BCC Fe. This implies that the elemental $\omega \mathrm{Fe}$ should be difficult to be formed. In experiments, however, the nanometer-size $\omega$ structure was observed in steel $[1,2]$. The largest difference between elemental $\mathrm{Fe}$ and steel may be the presence of $\mathrm{C}$ atoms, but little has been known about effects of the $\mathrm{C}$ atoms on the stability of the $\omega$ structure in steel.

Here we report a first-principles study on the stability of the $\omega$ structure in steel with special interests in effects

Email addresses: ikeda.yuji.6m@kyoto-u.ac.jp (Yuji Ikeda), tanaka@cms.mtl.kyoto-u.ac.jp (Isao Tanaka) of interstitial $\mathrm{C}$ atoms. Four different magnetic states of the $\omega$ structure investigated in our previous study [5] (see Fig. 1(c) in Ref. [5] for notations of the magnetic states) are focused on. Possible interstitial sites for $\mathrm{C}$ atoms in the $\omega$ structure are first systematically searched, and then the energy of the $\omega$ structure is compared with that of the FM BCC at several C concentrations using supercell models. Finally, mechanical stability of the $\omega$ structure is analyzed based on phonon frequencies at the $\Gamma$ point of the supercell models.

\section{Computational details}

The $\omega$ structure belongs to the hexagonal crystal system, and hence its primitive unit cell is specified by two lattice constants $a_{\omega}$ and $c_{\omega}$. The $\omega$ structure has three atoms inside the primitive unit cell, and their positions are $(0,0,0),(2 / 3,1 / 3,1 / 2)$, and $(1 / 3,2 / 3,1 / 2)$ in fractional coordinates. The $\omega$ structure can actually be obtained from the BCC structure by repeating to collapse a pair of neighboring $\{111\}$ planes and to hold the next plane unaltered.

Four magnetic states, which were focused on in our previous study [5], were investigated for the $\omega$ structure. The FM BCC structure and cementite $\mathrm{Fe}_{3} \mathrm{C}_{1}$ were also calculated for comparison. The $\omega$-based BCC unit cell (see Fig. 1(b) in Ref. [5] for details) was used for the calculation of the FM BCC structure so that computational conditions for the $\omega$ and the BCC structures are as similar to each other as possible.

To systematically search possible interstitial sites for $\mathrm{C}$ atoms in the $\omega$ structure in $\mathrm{Fe}$, we used the $\omega \mathrm{Fe}_{24} \mathrm{C}_{1}$ model composed of the $2 \times 2 \times 2$ supercell of the primitive $\omega$ unit 
cell for Fe and an interstitial $\mathrm{C}$ atom. The possible interstitial sites were searched in the following procedure. First, we divided a primitive $\omega$ unit cell into the $6 \times 6 \times 4$ mesh and put a $\mathrm{C}$ atom on the mesh points that are symmetrically inequivalent to each other. The numbers of the inequivalent points were 30 for the ++- magnetic state and 21 for the other magnetic states. Then, we optimized lattice parameters and internal atomic positions of the structures of the supercell models. Some of the supercell models showed atomic positions largely deviated from those of the initial $\omega$ structure after the structural optimization. These models were excluded from further consideration. Similarly, some models were excluded because the initially given magnetic state was broken after the structural optimization.

Energies of the models were calculated based on the plane-wave basis projector augmented wave method [6] in the framework of density-functional theory within the generalized gradient approximation of the Perdew-BurkeErnzerhof form [7] as implemented in the VASP code $[8,9,10]$. A plane-wave energy cutoff of $400 \mathrm{eV}$ was used. The Brillouin zones were sampled by the $\Gamma$-centered $12 \times 12 \times 18$ mesh per primitive $\omega$ unit cell, and the Methfessel-Paxton scheme [11] with a smearing width of $0.4 \mathrm{eV}$ was employed. Total energies were minimized until the energy convergences to be less than $10^{-8} \mathrm{eV}$. Lattice parameters and internal atomic positions were optimized under zero external stress.

Phonon frequencies were investigated within the harmonic approximation for a lattice Hamiltonian using the finite-displacement method. Atomic displacements of $0.01 \AA$ were used to obtain the second-order force constants. Phonon modes at the $\Gamma$ point of the supercell models were calculated to investigate their mechanical stability. When there are phonon modes with imaginary frequencies, the structure is considered to be mechanically unstable. The PHONOPY code $[12,13]$ was used for these phonon calculations.

\section{Results and discussion}

In our previous study [5], we made a systematic firstprinciples study on thermodynamical and mechanical stability of the $\omega$ structure in 27 transition elements ( $\mathrm{Sc}$ to $\mathrm{Cu}, \mathrm{Y}$ to $\mathrm{Ag}$, and $\mathrm{Lu}$ to $\mathrm{Au}$ ). Only the $\omega$ structures of the group 4 elements (Ti, Zr, and Hf), the group 7 elements (Mn, Tc, and Re), and Fe were found to be mechanically stable in their lowest-energy magnetic states. For the elemental $\omega \mathrm{Fe}$, the +- magnetic state was the lowest in energy among the investigated magnetic states. It was, however, $170 \mathrm{meV} /$ atom higher in energy than the FM BCC Fe. This implies that the elemental $\omega$ Fe is difficult to be formed from the viewpoint of thermodynamical stability. In the following, we investigate whether the presence of interstitial $\mathrm{C}$ atoms stabilizes the $\omega$ structure in Fe.

First, we search possible interstitial sites for $\mathrm{C}$ atoms in the $\omega$ structure based on the systematic procedure de-
Table 1: Calculated energies of the $\omega \mathrm{Fe}_{24} \mathrm{C}_{1}$ models in $\mathrm{meV} /(\mathrm{Fe}$ atom). The energies are relative to that for the FM BCC $\mathrm{Fe}_{24} \mathrm{C}_{1}$ models where the $\mathrm{C}$ atom is located at an octahedral site. The first column shows the initial position of the interstitial $\mathrm{C}$ atom in fractional coordinates for a primitive $\omega$ unit cell in the supercell models before the structural optimization. "NA" indicates that the corresponding structure could not be obtained because the optimized structure was largely deviated from the initial $\omega$ structure and/or because the initially given magnetic state was broken during the structural optimization.

\begin{tabular}{ccccc}
\hline Initial position of C & FM & ++- & +-- & NM \\
\hline$(0,1 / 2,0)$ & 182 & 212 & 182 & 276 \\
$(1 / 6,1 / 3,0)$ & 224 & NA & 196 & 299 \\
$(1 / 6,1 / 3,1 / 2)$ & 207 & NA & 214 & 325 \\
$(0,1 / 6,1 / 2)$ & 216 & NA & 223 & 330 \\
$(0,1 / 2,1 / 2)$ & 251 & NA & 274 & 389 \\
$(1 / 3,2 / 3,0)$ & NA & 365 & NA & 417 \\
$(0, \quad 0,1 / 2)$ & NA & NA & 349 & 428 \\
\hline
\end{tabular}

(a)

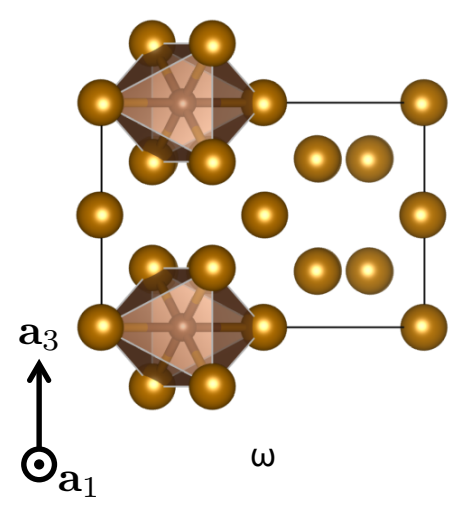

(b)

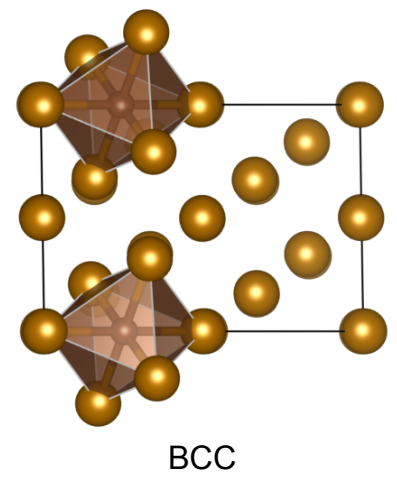

Figure 1: (Color online) (a) Structure of the $\omega \mathrm{Fe}_{24} \mathrm{C}_{1}$ model where the $\mathrm{C}$ atom is located at the octahedral site. (b) Structure of the BCC $\mathrm{Fe}_{24} \mathrm{C}_{1}$ model where the $\mathrm{C}$ atom is located at the octahedral site. Gold spheres represent Fe atoms, and grey spheres inside the octahedra represent $\mathrm{C}$ atoms. $\mathbf{a}_{1}$ and $\mathbf{a}_{3}$ denote two lattice vectors of the models. Visualization is performed using the VESTA code [14]. 


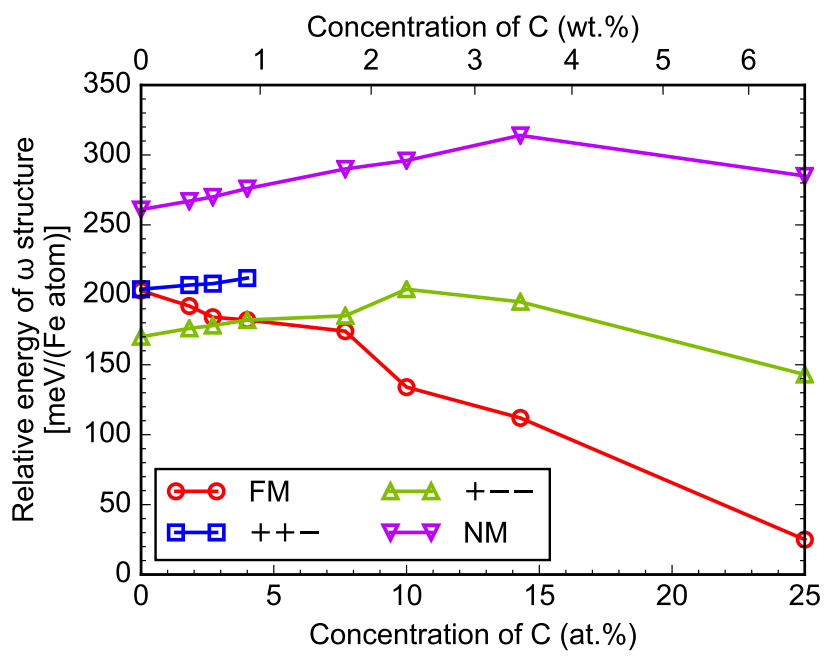

Figure 2: (Color online) Calculated energies of the $\omega \mathrm{Fe}_{n} \mathrm{C}_{1}$ models where the $\mathrm{C}$ atom is located at an octahedral site with respect to the concentration of $\mathrm{C}$. The energies are relative to that of the FM BCC $\mathrm{Fe}_{n} \mathrm{C}_{1}$ models where the $\mathrm{C}$ atom is located at an octahedral site. Lines are guides for the eyes. Note that the energy of the ++$\omega$ structure cannot be obtained above 4 at.\% C because magnetic states of the optimized structures were broken during the structural optimization.

scribed above. Table 1 summarizes calculated energies of the obtained $\omega \mathrm{Fe}_{24} \mathrm{C}_{1}$ models. Calculations from the inequivalent initial positions of the $\mathrm{C}$ atom converged to seven positions as listed in Table 1 . When the initial position of the $\mathrm{C}$ atom is $(0,1 / 2,0)$ in fractional coordinates for a primitive $\omega$ unit cell, the energy is the lowest among those of the interstitial sites for all the magnetic states. The $(0,1 / 2,0)$ interstitial site can be referred to as the octahedral site of the $\omega$ structure, as shown in Fig. 1(a). The octahedral site of the $\omega$ structure is actually similar to that of the BCC structure, as shown in Fig. 1(b). It has been well investigated that $\mathrm{C}$ atoms favor the octahedral site in the BCC Fe [15]. The present calculations reveal good correspondence of the favorable interstitial sites between the two crystal structures.

To investigate dependence of energies on the concentration of $\mathrm{C}$ atoms, we use the $1 \times 1 \times 1,1 \times 1 \times 2,1 \times 1 \times 3$, $2 \times 2 \times 1,2 \times 2 \times 2,2 \times 2 \times 3$, and $3 \times 3 \times 2$ supercells of the primitive $\omega$ unit cell with a $\mathrm{C}$ atom at an octahedral site. Hereafter supercell models incorporating a $\mathrm{C}$ atom are collectively referred to as $\mathrm{Fe}_{n} \mathrm{C}_{1}(n=3,6,9,12,24,36,54)$, where $n$ indicates the number of Fe atoms in the supercell models. Energies of the FM BCC $\mathrm{Fe}_{n} \mathrm{C}_{1}$ where the $\mathrm{C}$ atom is located at an octahedral site are also investigated using the corresponding supercells for comparison. Figure 2 shows the calculated energies of the $\omega \mathrm{Fe}_{n} \mathrm{C}_{1}$ relative to those of the FM BCC $\mathrm{Fe}_{n} \mathrm{C}_{1}$. The $\omega$ structure is higher in energy than the FM BCC up to 25 at. $\% \mathrm{C}$ for all the magnetic states. This result indicates that the $\omega$ structure is thermodynamically less favorable than the FM BCC even
Table 2: Numbers of imaginary phonon modes at the $\Gamma$ point of the $\mathrm{FM}$ and $+--\omega \mathrm{Fe}_{n} \mathrm{C}_{1}$ where the $\mathrm{C}$ atom is located at an octahedral site.

\begin{tabular}{cccc}
\hline & Supercell size & $\mathrm{FM}$ & +-- \\
\hline $\mathrm{Fe}_{3} \mathrm{C}_{1}$ & $1 \times 1 \times 1$ & 0 & 0 \\
$\mathrm{Fe}_{6} \mathrm{C}_{1}$ & $1 \times 1 \times 2$ & 1 & 1 \\
$\mathrm{Fe}_{9} \mathrm{C}_{1}$ & $1 \times 1 \times 3$ & 2 & 1 \\
$\mathrm{Fe}_{12} \mathrm{C}_{1}$ & $2 \times 2 \times 1$ & 6 & 3 \\
$\mathrm{Fe}_{24} \mathrm{C}_{1}$ & $2 \times 2 \times 2$ & 7 & 2 \\
$\mathrm{Fe}_{36} \mathrm{C}_{1}$ & $2 \times 2 \times 3$ & 8 & 2 \\
$\mathrm{Fe}_{54} \mathrm{C}_{1}$ & $3 \times 3 \times 2$ & 19 & 3 \\
$\mathrm{Fe}$ & & 1 & 0
\end{tabular}

when interstitial $\mathrm{C}$ atoms are incorporated. Furthermore, the relative energy of the $\omega$ structure below 14 at.\% C is higher than that of the elemental $\omega$ Fe for the ++- , +-- , and nonmagnetic (NM) states. This indicates that interstitial $\mathrm{C}$ atoms thermodynamically destabilize the $\omega$ structure in the,++-+-- , and NM states at these $\mathrm{C}$ concentrations compared with the FM BCC. For the FM $\omega$ structure, in contrast, the relative energy monotonously decreases as the $\mathrm{C}$ concentration increases. While the FM $\omega$ structure is $32 \mathrm{meV} /(\mathrm{Fe}$ atom) higher in energy than the $+--\omega$ when no interstitial $\mathrm{C}$ atoms are incorporated, the energy difference becomes smaller as the $\mathrm{C}$ concentration increases. Finally the FM $\omega$ structure becomes lower in energy than the $+--\omega$ above 4 at.\% $\mathrm{C}$.

Table 2 summarizes the number of imaginary phonon modes at the $\Gamma$ point of the $\mathrm{FM}$ and $+--\omega \mathrm{Fe}_{n} \mathrm{C}_{1}$ where the $\mathrm{C}$ atom is located at an octahedral site. Except for the $\omega \mathrm{Fe}_{3} \mathrm{C}_{1}$, which corresponds to 25 at. $\% \mathrm{C}$, all the models with interstitial $\mathrm{C}$ atoms have one or more imaginary phonon modes at the $\Gamma$ point and hence are mechanically unstable. Although the elemental $+--\omega$ Fe is mechanically stable [5], it becomes mechanically unstable once it incorporates interstitial $\mathrm{C}$ atoms unless the $\mathrm{C}$ concentration is 25 at.\%. This result indicates that the interstitial $\mathrm{C}$ atoms mechanically destabilize the $\omega$ structure in Fe. Although the $\omega \mathrm{Fe}_{3} \mathrm{C}_{1}$ shows no imaginary phonon modes at the $\Gamma$ point, the $\mathrm{FM} \omega \mathrm{Fe}_{3} \mathrm{C}_{1}$ is still $25 \mathrm{meV} /(\mathrm{Fe}$ atom $)$ higher in energy than the $\mathrm{FM} \mathrm{BCC} \mathrm{Fe}_{3} \mathrm{C}_{1}$ and hence is thermodynamically less favorable. Furthermore, it is well known that cementite $\mathrm{Fe}_{3} \mathrm{C}_{1}$ is thermodynamically much more favorable in such a high $\mathrm{C}$ concentration region. Our first-principles calculations actually show that the FM cementite $\mathrm{Fe}_{3} \mathrm{C}_{1}$ is 111 and $135 \mathrm{meV} /(\mathrm{Fe}$ atom) lower in energy than the FM BCC $\mathrm{Fe}_{3} \mathrm{C}_{1}$ and the $\mathrm{FM} \omega \mathrm{Fe}_{3} \mathrm{C}_{1}$, respectively.

The present calculations demonstrate that the $\omega$ structure is thermodynamically less favorable than the FM BCC irrespective of the $\mathrm{C}$ concentration up to 25 at.\%. Furthermore, the FM and the $+--\omega$ structures are mechanically unstable once they incorporate $\mathrm{C}$ atoms unless the $\mathrm{C}$ concentration is 25 at.\%. These results imply the instability of the $\omega$ structure in Fe-C alloys. We conclude that the $\omega$ structure is mostly unstable and cannot exist 
even as a metastable state in $\mathrm{Fe}-\mathrm{C}$ alloys. In experimental reports $[1,2]$, the $\omega$ structure was observed in steel as nanometer-size domains in the BCC structure or as nanometer-thick layers at twin boundaries of the BCC. Based on the present theoretical results, we suggest that the $\omega$ structure in steel is formed under special atomic constraints at twin boundaries or other interfaces. It is actually known that twin boundaries in metallic systems sometimes show special structures such as the $9 R$ structure in $\mathrm{Cu}$ and $\mathrm{Ag}[16,17]$. Since the $\omega$ structure acts as the transition state of a transition pathway between the BCC and the FCC structures, the $\omega$ structure in steel may be a kind of residue of the martensitic transformation. Another possible factor may be enrichment of solute elements other than interstitial $\mathrm{C}$ atoms in the nanometer-scale regions. The specimens used in the experiments actually contain $\mathrm{Si}, \mathrm{Mn}$, and/or $\mathrm{Cr}$ as well as $\mathrm{C}[1,2]$.

Finally we comment on theoretical calculations in Ref. [1]. Two points are inconsistent with the present results. First, the energy of the $\omega$ structure relative to that of the BCC structure is substantially different at 7.7 and 14.3 at.\% C between Ref. [1] and the present report. The authors in Ref. [1] reported that the relative energies are 142 and $78 \mathrm{meV} /(\mathrm{Fe}$ atom) at 7.7 and 14.3 at.\% C, respectively. In contrast, the present calculations show that the energies of the FM $\omega$ structure relative to those of the FM BCC are 174 and $112 \mathrm{meV} /(\mathrm{Fe}$ atom) at 7.7 and 14.3 at.\% C, respectively, which are much larger than the values in Ref. [1]. Since the authors in Ref. [1] do not refer to magnetic states of the $\omega$ structure incorporating $\mathrm{C}$ atoms, it is difficult to discuss possible reasons for the discrepancies. Note that the present calculations demonstrate that the FM $\omega$ structure is the lowest in energy among the magnetic states above 4 at.\% C, which was not reported in Ref. [1]. Second, the authors of Ref. [1] claimed that the $\mathrm{BCC} \mathrm{Fe}_{3} \mathrm{C}_{1}$ changed to the $\omega \mathrm{Fe}_{3} \mathrm{C}_{1}$ without energy barriers. They did not, however, show the computational details for this issue. Contrary to their results, our FM BCC $\mathrm{Fe}_{3} \mathrm{C}_{1}$ is mechanically stable and is $25 \mathrm{meV} /\left(\mathrm{Fe}\right.$ atom) lower in energy than the $\mathrm{FM} \omega \mathrm{Fe}_{3} \mathrm{C}_{1}$.

\section{Conclusion}

Stability of the $\omega$ structure in steel is investigated based on first-principles with special interests in effects of interstitial $\mathrm{C}$ atoms. The energy of the $\omega$ structure is compared with that of the FM BCC, and mechanical stability is analyzed based on phonon frequencies at the $\Gamma$ point of supercell models.

Possible interstitial sites for $\mathrm{C}$ atoms in the $\omega$ structure are systematically searched, and the octahedral site is found to be the most favorable among the interstitial sites. The $+--\omega \mathrm{Fe}_{n} \mathrm{C}_{1}$ where the $\mathrm{C}$ atom is located at an octahedral site is the lowest in energy among the magnetic states below 4 at.\% C, while the $\mathrm{FM} \omega \mathrm{Fe}_{n} \mathrm{C}_{1}$ is the lowest above this concentration.
Even when $\mathrm{C}$ atoms are incorporated in the $\omega$ structure, it is thermodynamically less favorable than the FM BCC. Furthermore, the FM and $+--\omega$ structures are mechanically unstable once they incorporate $\mathrm{C}$ atoms unless the $\mathrm{C}$ concentration is 25 at. $\%$. These results indicate that interstitial $\mathrm{C}$ atoms destabilize the $\omega$ structure in Fe-C alloys. It is concluded that the $\omega$ structure is mostly unstable and cannot exist even as a metastable state in Fe-C alloys. The $\omega$ structure in steel observed in experiments may be stabilized under special atomic constraints at twin boundaries or other interfaces.

\section{Acknowledgments}

The authors thank D. Ping for providing valuable information on the observation of the $\omega$ structure in steel. Funding by the Ministry of Education, Culture, Sports, Science and Technology (MEXT), Japan, through Elements Strategy Initiative for Structural Materials (ESISM) of Kyoto University, is gratefully acknowledged.

\section{References}

\section{References}

[1] D. Ping, W. Geng, Mater. Chem. Phys. 139 (2013) 830-835.

[2] T. Liu, D. Zhang, Q. Liu, Y. Zheng, Y. Su, X. Zhao, J. Yin, M. Song, D. Ping, Sci. Rep. 5 (2015) 15331.

[3] A. Togo, I. Tanaka, Phys. Rev. B 87 (2013) 184104.

[4] Y. Ikeda, A. Seko, A. Togo, I. Tanaka, Phys. Rev. B 90 (2014) 134106.

[5] Y. Ikeda, I. Tanaka, 2016.

[6] P. E. Blöchl, Phys. Rev. B 50 (1994) 17953-17979.

[7] J. P. Perdew, K. Burke, M. Ernzerhof, Phys. Rev. Lett. 77 (1996) 3865-3868.

[8] G. Kresse, J. Non-Cryst. Solids 192-193 (1995) 222-229.

[9] G. Kresse, J. Furthmüller, Comput. Mater. Sci. 6 (1996) 15-50.

[10] G. Kresse, D. Joubert, Phys. Rev. B 59 (1999) 1758-1775.

[11] M. Methfessel, A. T. Paxton, Phys. Rev. B 40 (1989) 3616-3621.

[12] A. Togo, I. Tanaka, Scr. Mater. 108 (2015) 1-5.

[13] A. Togo, F. Oba, I. Tanaka, Phys. Rev. B 78 (2008) 134106

[14] K. Momma, F. Izumi, J. Appl. Crystallogr. 44 (2011) 12721276 .

[15] D. E. Jiang, E. A. Carter, Phys. Rev. B 67 (2003) 214103.

[16] U. Wolf, F. Ernst, T. Muschik, M. W. Finnis, H. F. Fischmeister, Philos. Mag. A 66 (1992) 991-1016.

[17] F. Ernst, M. W. Finnis, D. Hofmann, T. Muschik, U. Schönberger, U. Wolf, M. Methfessel, Phys. Rev. Lett. 69 (1992) 620-623. 